\title{
An Observational Study Assessing Changes in Health and Functional Status in Patients with Chronic Obstructive Pulmonary Disease (COPD) During Therapy with Spiolto ${ }^{\circledR}$ Respimat $^{\circledR}$ in Everyday Clinical Practice: The Greek ELLACTO Study
}

Epaminondas Kosmas · Iraklis Titopoulos • Georgios Patentalakis •

Nikos Nikas

Received: March 8, 2021 / Accepted: April 13, 2021 / Published online: May 3, 2021

(c) The Author(s) 2021

\section{ABSTRACT}

Introduction: Patients with chronic obstructive pulmonary disease (COPD) often report deteriorated functional status and poor health-related quality of life, both core aspects of their overall health status. The study objective was to assess tiotropium/olodaterol Respimat ${ }^{\circledR}$ effects on health and functional status of COPD patients requiring long-acting dual bronchodilation treatment in the real world.

Methods: ELLACTO was an open-label, observational, prospective study conducted in Greece, measuring changes on health and functional status of COPD patients treated with a fixed dose of tiotropium/olodaterol Respimat ${ }^{\circledR}$ for approximately 6 weeks. The primary endpoint was "therapeutic success" defined as a $\geq 0.4$-point decrease in the Clinical COPD

\section{E. Kosmas}

Metropolitan Hospital, Athens, Greece

e-mail: ath.lung.inst@gmail.com

\section{Titopoulos}

European Interbalkan Medical Center, Thessaloniki, Greece

e-mail: ir.titop@otenet.gr

\section{G. Patentalakis $(\bowtie) \cdot$ N. Nikas}

Boehringer Ingelheim Ellas, 340, A. Syngrou

Avenue, 17673 Athens, Greece

e-mail: georgios.patentalakis@boehringer-

ingelheim.comN. Nikas

e-mail: nikos.nikas@boehringer-ingelheim.com
Questionnaire (CCQ) score at week 6. Secondary endpoints included absolute changes in the CCQ and the functional subscale CCQ-4 at week 6 , patient general condition measured by Physician's Global Evaluation (PGE) score at baseline and week 6 , patient satisfaction and preference with Respimat ${ }^{\circledR}$ device (assessed by the abbreviated Patient Satisfaction and Preference Questionnaire (PASAPQ)) at week 6 and treatment continuation with tiotropium/olodaterol Respimat ${ }^{\circledR}$ after the study.

Results: After approximately 6 weeks of treatment with tiotropium/olodaterol Respimat ${ }^{\circledR}$, therapeutic success was achieved by $64.3 \%$ of 1332 patients included in the final analysis [95\% confidence interval [CI] 62-67]. Mean [standard deviation (SD)] absolute changes in CCQ and CCQ-4 scores at week 6 were -0.63 (0.635) and -0.59 (0.714) points, respectively. Patient general condition improved and more than $77.4 \%$ of patients were satisfied or very satisfied with the use of the Respimat ${ }^{\circledR}$ device. Among patients previously using the HandiHaler $^{\circledR}$ device $(n=254), \quad 85.4 \%$ expressed a preference for Respimat ${ }^{\circledR}$ over $6.7 \%$ for HandiHaler $^{\circledR}$. Most patients (95.7\%) were willing to continue treatment with tiotropium/olodaterol Respimat ${ }^{\circledR}$ after the study.

Conclusions: Treatment with tiotropium/olodaterol Respimat ${ }^{\circledR}$ led to an improved health status in Greek COPD patients. Most patients also expressed a preference for the Respimat ${ }^{\circledR}$ 
device and willingness to continue treatment with it.

Trial registration: ClinicalTrials.gov NCT03419962.

Keywords: COPD; Dual bronchodilation; Functional status; Health status; Olodaterol; Tiotropium

\section{Key Summary Points}

Why carry out this study?

Patients with COPD often report deteriorated functional status and poor health-related quality of life, both core aspects of their overall health status.

Tiotropium/olodaterol fix-dose combination (FDC) improves health and functional status of patients suffering from chronic obstructive pulmonary disease (COPD).

The effects of tiotropium/olodaterol FDC administered via the Respimat device on health and functional status of COPD patients under real-life conditions in Greece has not yet been investigated.

\section{What was learned from the study?}

Tiotropium/olodaterol Respimat conferred a beneficial effect on self-reported health and functional status in a large group of Greek patients with COPD.

A concurrent improvement in patients' general condition was observed.

The majority of patients expressed satisfaction and preference for the Respimat device and a willingness to continue treatment with it after the end of the study.

\section{DIGITAL FEATURES}

This article is published with digital features, including a summary slide, to facilitate understanding of the article. To view digital features for this article go to https://doi.org/10.6084/ m9.figshare.14402465.

\section{INTRODUCTION}

COPD is a chronic inflammatory disease of the airways, characterized by persistent respiratory symptoms, progressive breathlessness, and poorly reversible airflow obstruction [1]. When the disease becomes aggravated, patients gradually suffer from deteriorated functional status and poor health-related quality of life. Impaired functional status has been documented as a predictor for an increase in exacerbation frequency, hospital admission, and mortality, with a reduction in physical activity level being one of the strongest predictors of mortality in patients with COPD [2]. Assessment of patientreported outcomes measuring the impact of the disease on day-to-day functioning, can therefore be as important as objective measurements of airflow limitation in determining the treatment benefit for patients with COPD [3].

Long-acting bronchodilators, such as longacting muscarinic antagonists (LAMAs) and long-acting $\beta_{2}$-agonists (LABAs), represent the cornerstone of maintenance therapy for COPD [4]. Monotherapy is usually not satisfactory for patients with more severe COPD [1], therefore, the Global initiative for chronic Obstructive Lung Disease (GOLD) guidelines currently recommend the use of LABAs and LAMAs in combination for the majority of COPD patients [5].

Tiotropium/olodaterol, formulated in the Respimat $^{\circledR}$ (Boehringer Ingelheim, Ingelheim am Rhein, Germany) soft-mist inhaler, is an inhaled fixed-dose combination (FDC) of the LAMA tiotropium and the LABA olodaterol approved in the European Union (EU) for the long-term maintenance treatment of COPD [6]. The efficacy of tiotropium/olodaterol in patients with COPD has been extensively evaluated in the $>16,000$ patient TOviTO ${ }^{\circledR}$ Phase III clinical trial program, one of the largest 
clinical development programs conducted in COPD. Tiotropium/olodaterol conferred significant improvements in pulmonary function, exacerbation rate and exercise capacity, as well as patient-reported outcomes (such as dyspnea), when compared with either placebo or its monocomponents [6-13].

Given that health status assessment is increasingly acknowledged as a key component of the overall assessment of treatment effectiveness in COPD, two large, controlled, randomized phase III trials within the ToviTO program-the pivotal TOnado and the OTEMTO trials [14, 15]—investigated the effect of tiotropium/olodaterol on health status. Results from these trials demonstrated significantly larger improvements in health status as assessed by the St. George's Respiratory Questionnaire (SGRQ) with tiotropium/olodaterol than with either placebo or monotherapy $[3,14,15]$.

Observational studies conducted across several countries over the recent years have also provided evidence of the positive effects of tiotropium/olodaterol on physical functioning [16-21], a core aspect of health status. Nevertheless, real-life data demonstrating a direct effect of the drug on the overall COPD-related health status are still scarce, whereas, to our knowledge, no data are available for the Greek population.

In this non-interventional study (NIS), we aimed to investigate the effectiveness of tiotropium/olodaterol Respimat in improving health and functional status of patients with COPD under real-life conditions in Greece. Further objectives were to assess patient satisfaction and preference with tiotropium/olodaterol delivered via the Respimat device, and to evaluate general patient condition.

\section{METHODS}

\section{Study Design}

ELLACTO was an open-label, non-interventional prospective study that assessed the effect of dual long-acting bronchodilation with tiotropium/olodaterol Respimat on health and functional status of COPD patients impaired in activity of daily living (ADL) in routine clinical practice in Greece. Patients were enrolled consecutively and followed over an observational period of approximately 6 weeks. Treatment with tiotropium/olodaterol Respimat was performed according to the local Summary of Product Characteristics (SmPC) and as per the routine medical practice in terms of patient procedures and assessment types. The decision to treat the patient with tiotropium/olodaterol Respimat had already been taken prior to study enrolment and was clearly separated from the physician's decision to include them in the study as per the non-interventional status of the study. Safety was assessed as per company and local regulations for NIS, based on collection of adverse drug reactions (ADR) and fatal adverse events (AEs) during the study period.

The study was conducted at 102 sites across Greece by 100 pulmonologists in private practice and two hospitals between March 2018 and April 2019. Practices were spread throughout Greece, including both urban and rural areas, to ensure a sample representative of the general COPD population. The study was conducted in compliance with the principles laid down in the Declaration of Helsinki and local regulatory requirements, as per the protocol, with approval obtained by the relevant Institutional Review Committees/Ethics Committees. All patients provided written informed consent before participating in the study. The trial is registered in clinicaltrials.gov under the identifier NCT03419962.

\section{Study Population}

Patients eligible to participate in the study were: females and males aged $\geq 40$ years, diagnosed with COPD requiring dual bronchodilation treatment with two long-acting bronchodilators (LAMA + LABA) in accordance with the GOLD 2017 guidelines regarding groups B to D [5], prescribed tiotropium/olodaterol Respimat according to the physician's discretion and in line with the approved SmPC and having provided written informed consent prior to study participation. Patients were excluded from the 
study if they met any of the contraindications to tiotropium/olodaterol Respimat according to the SmPC, had been treated with a LABA/LAMA combination (either free or fixed-dose) in the previous 6 weeks, or had already been on a LABA/LAMA combination treatment (either free or fixed-dose); patients previously treated with LABA or LAMA [with or without inhaled corticosteroids (ICS)] could be included in the study.

\section{Visits and Assessments}

Patient assessments were performed at baseline (Visit 1 ), and after 6 weeks ( \pm 10 days) of treatment (Visit 2). A telephone contact was also planned 2 weeks after Visit 1 to check for treatment compliance and safety issues. During Visit 1, data on patient's demographics, smoking history, history of COPD, including number of overall exacerbations and exacerbations leading to hospitalization within the previous 12 months, spirometric COPD severity based on GOLD guidelines [5], past (within 6 months prior to Visit 1) and present concomitant respiratory/COPD medications, other concomitant diseases/comorbidities and other concomitant medications were collected. Patient's general condition was assessed using the PGE scale, an ordinal eight-point scale in which the physician evaluated the patient's general condition as poor (1-2), satisfactory (3-4), good (5-6), or excellent (7-8) [20]. The severity of dyspnea was measured via the modified Medical Research Council Dyspnea scale (mMRC). The mMRC is an ordinal four-point scale (grades 0-4) based on degrees of various physical activities that precipitate dyspnea, with grade 4 representing the most severe category [22]. mMRC together with exacerbation history, and GOLD spirometric classification of airflow limitation (if available) were also used to automatically calculate the GOLD patient group (B, C or D) within the electronic Case Report form (eCRF). Patients were asked to complete the CCQ, a validated and reliable self-completed ten-item questionnaire comprising of three subdomains (i.e., symptoms, functional state and mental state), originally developed to measure the clinical health status in patients with COPD
[23]. Patients were requested to recall their experiences during the previous week with regards to their symptoms, functional and mental state, and to respond to each of the ten corresponding questions on a seven-point scale (ranging between $0=$ asymptomatic/no-limitation and $6=$ extremely symptomatic/totally limited). The overall clinical CCQ score was calculated by adding all scores together and dividing the sum by 10 . The score for the functional status subdomain CCQ-4 was calculated by adding the scores of the four questions pertinent to functional status and dividing the sum by 4 . Higher scores indicate a worse health status [24].

At Visit 2, assessments of PGE, CCQ, and CCQ-4 scores were repeated. Moreover, patient satisfaction and preference to the Respimat device were assessed using the PASAPQ. PASAPQ is a multi-item practical, valid, reliable, and responsive measure of respiratory inhalation device satisfaction and preference designed to be easily understood and administered to patients with asthma and COPD. An important advantage of PASAPQ lies also in the fact that it is device- and treatment-independent, making it widely applicable to respiratory treatments [25]. In this study, an abbreviated, non-validated version of PASAPQ was utilized; in part 1 , patients were requested to better describe their satisfaction level concerning three inhaler attributes (feeling that the inhaled dose goes into the lungs, inhaler works reliably, and ease of inhaling a dose from the inhaler) by answering three relevant questions on a sevenpoint ordinal scale ranging from very dissatisfied to very satisfied. Part 2 assessed patient preference for Respimat versus HandiHaler ${ }^{\circledR}$ inhaler device and was applied only to those patients that had used tiotropium HandiHaler before the study. In part 2, patients were requested to describe their preference (selection between devices, as well as a "no preference" option) and willingness (measured on scale of 0 -not willing to 100 -definitely willing) to continue with each inhaler device in the future [26]. Whether patients were willing to continue treatment with Respimat after the study was also recorded at this visit based on a "Yes/No" question. Finally, changes related to 
respiratory/COPD medications, other concomitant diseases and medications as well as treatment discontinuations were recorded.

\section{Endpoints}

The primary efficacy endpoint of the study was achievement of "therapeutic success" defined as $\mathrm{a} \geq 0.4$-point decrease in the CCQ score between Visit 1 and Visit 2. As already known, 0.4 points is the well-accepted Minimal Clinically Important Difference (MCID) in the CCQ [27]. Secondary efficacy endpoints included absolute changes in CCQ and CCQ-4 scores from Visit 1 to Visit 2, patient's general condition as assessed by the PGE score at Visit 1 and Visit 2, patient's satisfaction with Respimat at Visit 2 (PASAPQ Part 1) and preference of Respimat vs. HandiHaler (PASAPQ Part 2)—only for those patients that had used Spiriva HandiHaler before the study-and, finally, the proportion (\%) of patients willing to continue treatment with Respimat following study completion.

\section{Statistical Analysis}

All enrolled patients receiving at least one documented administration of tiotropium/olodaterol Respimat were included in the Treated Set (TS). Safety and demographics/baseline characteristics were analyzed in the TS. Patients of the TS with a CCQ score available at both visit 1 and visit 2 constituted the full analysis set (FAS). All analyses related to primary and secondary endpoints were carried out in the FAS. In this observational study, all variables were analyzed in an exploratory manner using descriptive statistics. Summary statistics consisting of frequency tables were used for categorical variables. For continuous variables, descriptive statistics (number of available observations $[n]$, mean, SD) were tabulated unless specified otherwise. For the primary endpoint, the percentage of patients with therapeutic success was calculated together with the 95\% CI. Summary statistics were provided for changes in CCQ score, and the number and percentage of patients within each scale category were calculated for general patient condition and patient satisfaction with the Respimat device. Missing data were not imputed but were analyzed as missing. Statistical analysis and generation of tables and figures were performed using the Statistical Analysis System $\left(\mathrm{SAS}^{\circledR}\right)$ Version 9.2 or higher.

\section{RESULTS}

A total of 1363 patients were screened between March 19, 2018 and November 9, 2018, of whom 1360 were enrolled in the study. Of these, 1359 patients $(99.7 \%)$ were included in the TS and 1332 (97.7\%) in the FAS (Fig. 1). Mean time between treatment initiation and end of observation or discontinuation, whichever came first, was 49 days in the TS.

\section{Baseline Demographics and Disease Characteristics}

Baseline demographics and disease characteristics of patients in the TS population are presented in Table 1. Based on GOLD 2017 classification [5], most patients (62.2\%) were classified in group B, $21.3 \%$ in group C, and $16.1 \%$ in group D. Of the patients reporting symptoms of breathlessness (94.6\%), the majority were classified as mMRC grade 2 $(43.3 \%)$ and grade $3(22.7 \%)$. Most patients (61.8\%) had experienced at least one episode of COPD exacerbation in the 12 months prior to the study, with $7.7 \%$ of patients being hospitalized due to a COPD exacerbation. The majority of patients (62.4\%) had already received treatment for their COPD in the previous 6 months prior to study initiation (Table 2). Moreover, $26.7 \%$ of patients had an additional clinical diagnosis other than COPD, with cardiovascular diseases being the most common comorbid condition, and $25.6 \%$ of patients were receiving at least one concomitant medication other than respiratory drugs, mostly related to the above-mentioned diseases (Table 2). 


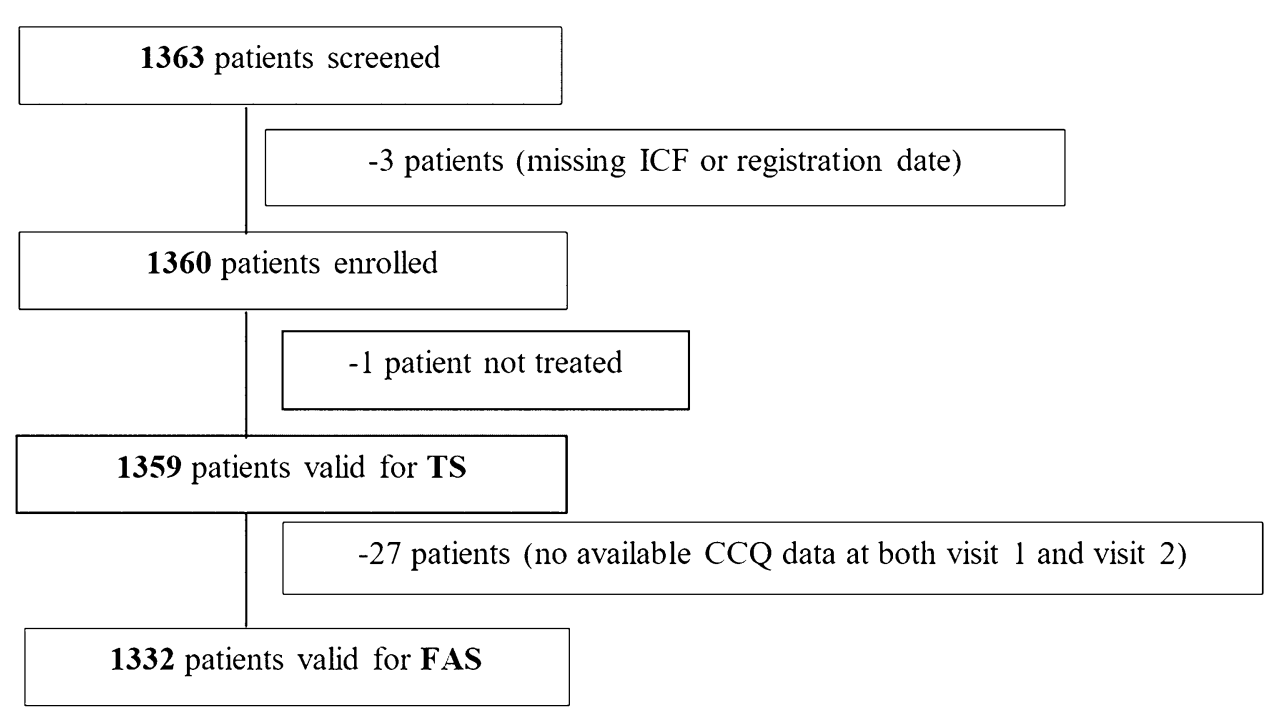

Fig. 1 Patient flow chart. CCQ Clinical COPD Questionnaire, FAS full analysis set, TS Treated Set

\section{Health and Functional Status}

After approximately 6 weeks on treatment with tiotropium/olodaterol Respimat, the primary efficacy endpoint of therapeutic success, i.e., a $\geq 0.4$-point decrease in the CCQ score between baseline (Visit 1) and 6 weeks (Visit 2), was achieved by $64.3 \%$ [95\% CI 62-67] of patients (FAS) (Fig. 2). The mean absolute change in the CCQ score (SD) between visit 1 and visit 2 was -0.63 (0.635). The mean absolute change in the corresponding score of the functional status subdomain of CCQ (CCQ-4) was - 0.59 (0.714).

A prespecified subgroup analysis of therapeutic success after 6 weeks for patients who were either maintenance-naïve (no LAMA or LABA or LABA + ICS within 6 months prior to study initiation) or had been already treated with LAMA or LABA or LABA + ICS was also performed, with corresponding rates of $66.6 \%$ $\left[\begin{array}{lll}95 \% & \text { CI } & 62.7-70.4\end{array}\right]$ and $62.5 \% \quad[95 \%$ CI 58.8-66.0]. According to a post hoc subgroup analysis of patients stratified by GOLD spirometric severity (stage 2 vs. $3 / 4$ ), success rate was $67.6 \%$ for grade 2 and 67.3 for grade $3 / 4$, whereas when stratified by GOLD patient group (group B vs. group C vs. group D), group D demonstrated the highest success rate $[77 \%$; 95\% CI 70.8-82.5], followed by group B [63.2\%;
95\% CI 59.8-66.5] and group C [58.2\%; 95\% CI 52.2-64.0].

When mean (SD) absolute changes in CCQ and CCQ-4 scores from visit 1 to visit 2 were also analyzed based on GOLD spirometric severity (stage 2 vs. 3 vs. 4), mean (SD) absolute changes in the CCQ score were - $0.63(0.576)$, $-0.70(0.724),-0.76(0.747)$, for stage 2,3 , and 4 , respectively, and the mean (SD) absolute changes in the CCQ-4 score were - $0.58(0.649)$, $-0.71(0.761)$, and $-0.73(0.872)$, for stage 2,3 , and 4, respectively. Upon stratification by GOLD group, the mean (SD) absolute change of CCQ score was - $0.86(0.696)$ for group D, followed by -0.59 (0.697) for group $C$ and -0.58 (0.580) for group B. Correspondingly, the mean (SD) absolute changes of CCQ-4 score for GOLD patient groups B, C, D were $-0.54(0.680)$, $-0.56(0.720)$ and $-0.85(0.775)$, respectively.

\section{General Patient Condition}

The patients' general condition, as assessed by their PGE score, showed a clear overall improvement from Visit 1 to Visit 2, with a shift from lower to higher PGE classes being observed (Fig. 3). Moreover, among patients with "poor", "satisfactory", and "good" general condition at Visit $1,80.4,68.2$, and $31.2 \%$ of them, 
Table 1 Baseline demographics and disease characteristics (TS; $N=1359$ )

Gender

$$
\text { Male, } n \text { (\%) }
$$

Age, years

$$
\text { Mean (SD) }
$$

Age at start of COPD, years

$N=1355$

Mean (SD)

$62.9(10.43)$

Smoking status, $n$ (\%)

Current smoker

$582(42.8)$

Ex-smoker

$674(49.6)$

Non-smoker

Missing

$101(7.4)$

$2(0.1)$

Pack years

$N$

1256

Mean (SD)

$52.4(27.84)$

Spirometric COPD severity, $n$ (\%)

Stage GOLD 1

$140(10.3)$

Stage GOLD 2

$730(53.7)$

Stage GOLD 3

$329(24.2)$

Stage GOLD 4

$107(7.9)$

Not performed

$49(3.6)$

Missing

$4(0.3)$

COPD GOLD group, $n$ (\%)

$\mathrm{B}$

$845(62.2)$

C

289 (21.3)

D

$219(16.1)$

Missing

$6(0.4)$

mMRC score, $n(\%)$

\begin{tabular}{ll}
0 & $60(4.4)$ \\
1 & $337(24.8)$ \\
2 & $589(43.3)$ \\
3 & $308(22.7)$ \\
4 & $47(3.5)$ \\
\hline
\end{tabular}

Table 1 continued

\begin{tabular}{lc}
\hline Missing & $18(1.3)$ \\
\hline$C O P D$ Chronic Obstructive Pulmonary
\end{tabular}

COPD Chronic Obstructive Pulmonary Disease, GOLD Global initiative for chronic Obstructive Lung Disease, $m M R C$ modified Medical Research Council, $S D$ standard deviation

respectively, showed an improvement of their PGE class at Visit 2.

\section{Patient Satisfaction and Preference to Respimat Device}

With regards to patient satisfaction with the use of Respimat as assessed by the abbreviated PASAPQ - Part 1 at the end of the study (Visit 2), the vast majority of patients (more than $77.4 \%$ ) reported to be "satisfied" or "very satisfied" in terms of all three performance attributes of device (Fig. 4).

At the end of the study, among patients who had used HandiHaler prior to the study $(n=254)$, the majority $(85.4 \%)$ showed a preference according to the PASAPQ Part 2 for Respimat device versus $6.7 \%$ that would prefer to use HandiHaler, while $7.9 \%$ reported no preference between devices (Fig. 5). Moreover, the mean (SD) rating for willingness to continue using the device after the study was 79.8 (21.19) for Respimat as compared to 32.3 (27.06) for HandiHaler, on a scale of $0-100$ where $0=$ "not willing" and $100=$ "definitely willing". Finally, the vast majority $(95.7 \%)$ of treated patients reported that they were willing to continue treatment with Respimat after the study.

\section{Safety}

Overall, four (0.3\%) patients reported any $\mathrm{AE}$ during the study, of whom two developed a gastrointestinal disorder (dry mouth) and one developed palpitations and were classified as ADRs. None of the ADRs led to drug discontinuation or required any action, and all recovered. 
Table 2 Concomitant diseases and medications at baseline (TS; $N=1359$ )

Concomitant diseases, $n(\%)$

Cardiovascular disorders

$268(19.7)$

Endocrine metabolism and nutrition disorders

$124(9.1)$

Central nervous system disorders

Gastrointestinal disorders

16 (1.2)

Others

Concomitant medications, $n$ (\%)

Beta-blockers

Other antihypertensives

Antithrombotics

Lipid-modifying agents

Blood glucose lowering agents

Previous COPD therapies (taken within the last 6 months prior to study treatment)
$\mathrm{LABA}+\mathrm{ICS}$
Salmeterol + fluticasone
$149(11.0)$
Formoterol + budesonide
$80(5.9)$
Formoterol + beclomethasone
$56(4.1)$
Other, not specified
$260(19.2)$
LAMA or ICS
Tiotropium bromide
Glycopyrronium bromide
Budesonide
Aclidinium bromide
Other
Other drugs for obstructive airway diseases
$44(3.2)$

ICS inhaled corticosteroid, $L A B A$ long-acting $\beta 2$-agonist, $L A M A$ long-acting muscarinic antagonist

No serious ADR or fatal serious AE (SAE) was reported. One SAE, namely COPD exacerbation, which led to hospitalization was reported, but was not considered drug-related by the physician.

\section{DISCUSSION}

This "real-life" observational study provides new data to the current literature in support of a potential beneficial effect of tiotropium/olodaterol Respimat on self-reported health and functional status in a large and heterogeneous group of Greek patients with COPD. The majority of included COPD patients receiving tiotropium/olodaterol Respimat for 


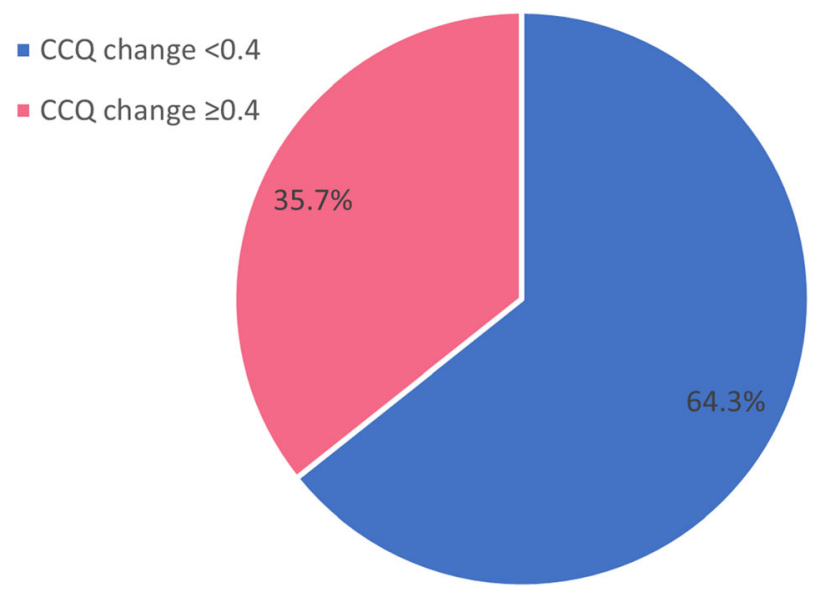

Fig. 2 Therapeutic success at Visit 2 (FAS; $N=1332$ ). CCQ Clinical COPD Questionnaire

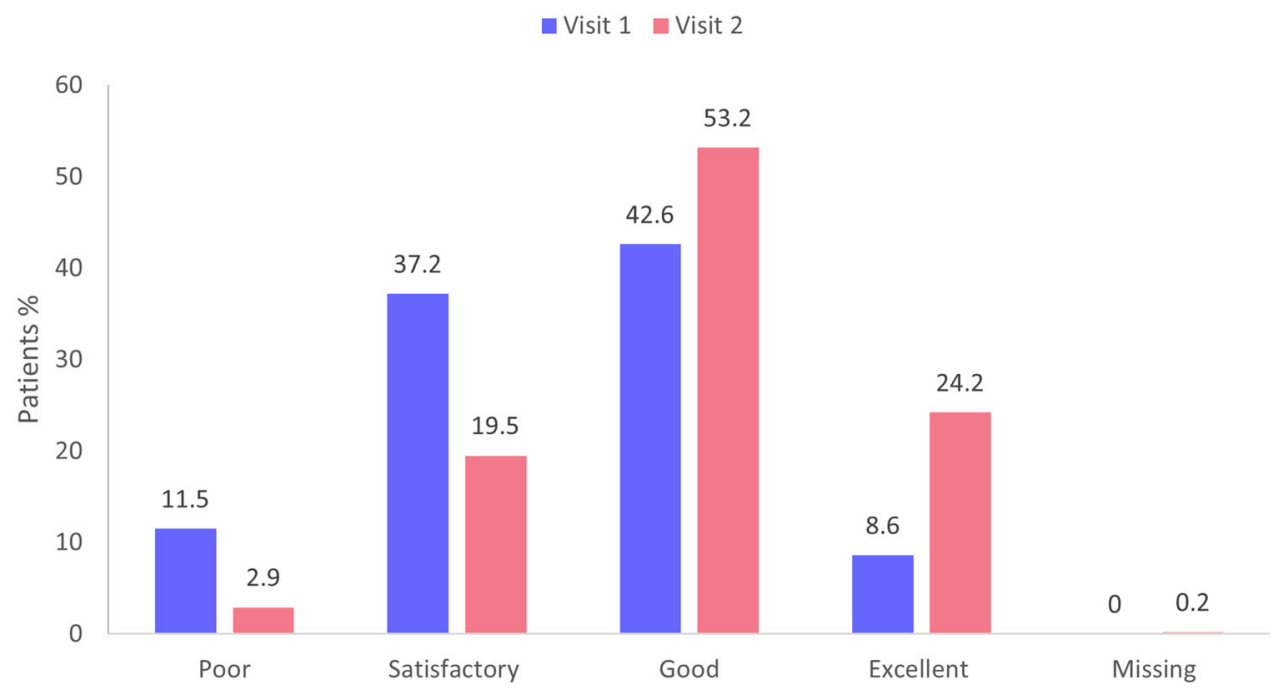

Fig. 3 PGE scores at Visit 1 and Visit 2 (FAS; $N=1332$ ). PGE Physician's Global Evaluation

approximately 6 weeks demonstrated a clinically meaningful improvement in their health status, defined as a decrease in the CCQ score of at least 0.4 points [27]. Not surprisingly, subgroup analyses using the GOLD 2017 classification criteria demonstrated that the proportion of patients achieving therapeutic success increased with increasing symptom severity, with a greater proportion of "responders" to tiotropium/olodaterol Respimat among GOLD D patients, followed by GOLD B and C, but was irrespective of airflow limitation severity, with similar proportions of "responders" among patients with moderate (GOLD 2) and severe/very severe disease (GOLD $3 / 4$ ). Moreover, health status, including functional status, was improved overall during the 6-week study period, as shown by corresponding decreases in the mean CCQ and the CCQ functional subdomain. These beneficial effects further translated into a concurrent improvement in patients' general condition, as demonstrated by an improvement in the distribution of physician-completed PGE scale scores, indicating that patients were generally in a healthier condition at 6 weeks compared to baseline. 


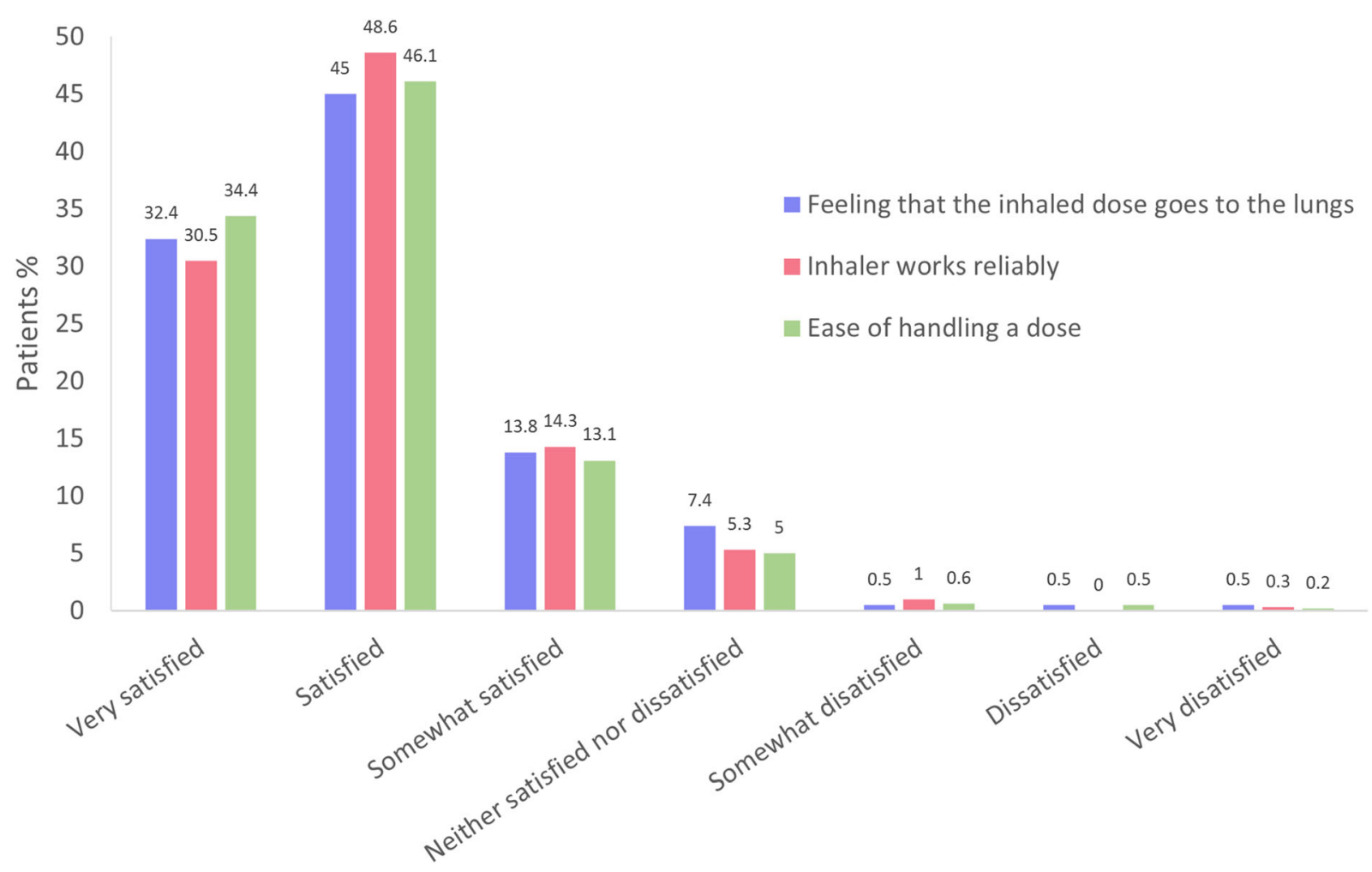

Fig. 4 Patient satisfaction with the Respimat inhaler in the abbreviated PASAPQ-Part 1 at Visit 2 (FAS; $N=1332$ ). $P A S A P Q$ Patient Satisfaction and Preference Questionnaire

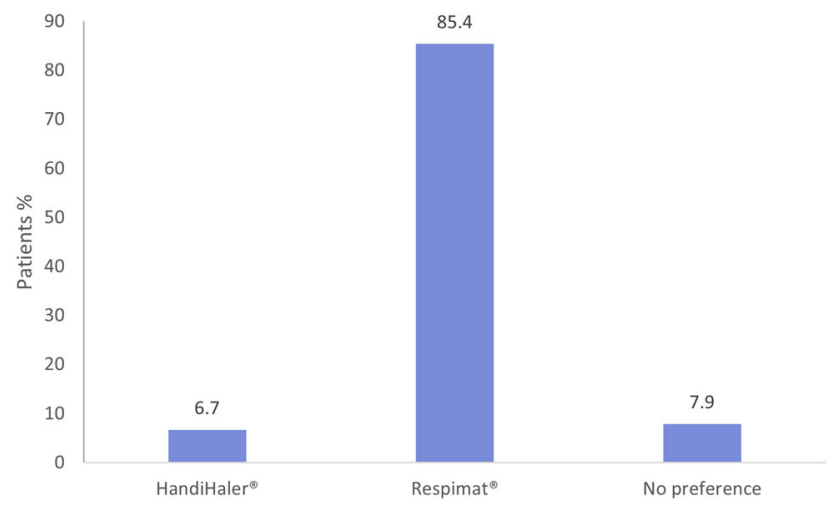

Fig. 5 Proportion of patients indicating preference for Respimat and HandiHaler inhalers in the abbreviated PASAPQ-Part 2 at Visit 2 (HandiHaler users switching

Our study complements the results of a number of large, observational studies of a similar design and of patient populations with similar characteristics previously conducted in other countries. These studies also demonstrated that in a typical outpatient COPD to the Respimat inhaler, $N=254)$. PASAPQ Patient Satisfaction and Preference Questionnaire

population, tiotropium/olodaterol Respimat confers a clinically meaningful improvement in self-reported physical functioning as assessed by the SF-36 Physical Functioning Subscale PF-10 following 6 weeks of treatment $[18,19,21]$. 
One major advantage of our study over the above-mentioned observational studies lies in the utilization of the CCQ questionnaire instead of the PF-10. CCQ is a well-known, validated health status questionnaire with widespread use in clinical practice [24], also currently included in the GOLD guidelines [5]. Health status is increasingly recognized as an important outcome measure in the setting of COPD, reflecting patients' perspective on the impact of their disease on symptom burden, functional capacity, and psychosocial functioning. To this end, CCQ could be used to successfully evaluate these three subdomains. The CCQ can also be used to evaluate the adequacy of clinical management in primary care and to further predict exacerbations and mortality in patients suffering from COPD [23]. Indeed, our study extends the previously reported findings by demonstrating that tiotropium/olodaterol Respimat has a beneficial effect not only in patients' physical functioning (a potential surrogate for health status) but also in the overall health status of patients with COPD. To our knowledge, this study, together with a recent multinational study conducted by Valipour and colleagues [22] are the first observational studies assessing the effectiveness of tiotropium/olodaterol Respimat in improving the overall health status of COPD, both demonstrating consistent results in terms of clinically meaningful improvements on health status over a similar treatment period [22].

Another important outcome assessed by this study was patient satisfaction with Respimat device. The vast majority of patients $(\geq 77.4 \%)$ were either "satisfied" or "very satisfied" with the use of the device, in all three attributes of its performance assessed, a fact that was further reflected by the high percentage of those willing to continue treatment after the end of the observation period $(95.7 \%)$. These results are in line with those of other observational studies, in which high levels of satisfaction with the use of Respimat were also reported by most patients [17-20]. Another interesting finding with respect to the use of Respimat was the reported preference in favor of Respimat when compared with previous use of HandiHaler, with the great majority of patients $(85.4 \%)$ preferring
Respimat. These results corroborate a small survey, which also demonstrated that patients with COPD seemed to consistently prefer Respimat over HandiHaler. Notably, the results of the latter study were extended by longer-term observation data up to approximately $2-3$ years, showing that the preference for Respimat increased with continued use over time [28]. Accordingly, in a number of clinical studies assessing patient satisfaction and preference that compared Respimat with other devices in patients with COPD, the Respimat device was consistently shown to be well accepted, largely due to its inhalation and handling characteristics $[26,29]$.

Given the potential impact that patients' experience with their inhalers may have on their adherence to treatment and hence longterm clinical outcomes, patient satisfaction and preference with inhaler devices have attracted increasing attention in the recent years [26]. Indicatively, a large, multinational, cross-sectional, survey demonstrated that satisfaction with the inhaler device was significantly associated with treatment compliance, with further significant associations between increased treatment compliance and reduction in exacerbations, as well as a direct association between increased patient satisfaction and less frequent exacerbations [16].

Taken together, satisfaction with the mode of treatment administration, combined with an improved health status and general condition, may all contribute to increased adherence to the prescribed medications, ultimately optimizing clinical outcomes.

Potential limitations of this study are mainly associated with its observational nature. Such limitations include the lack of a control group, potential risk for selection bias, mitigated though by consecutive patient enrolment, as well as the apparent presence of comorbidities and concomitant use of medications other than respiratory drugs that may act as confounders. Moreover, as self-reported questionnaires were utilized to evaluate the patient's health status and general condition, it is important to be aware of the potential bias related to such subjective measures. In general, patient-reported improvement seems to be overestimated 
compared to improvement measured objectively. On the one hand, self-reported measures successfully provide unique information of how patients feel and function, but on the other hand, there are trade-offs including problems of stability and interpretability [30]. It should also be also that no objective assessment, e.g., lung function, was performed to correlate any potential improvements in airway physiology with the self-reported benefits in health status and general condition. Another limitation associated with this study was the absence of a patient medication diary, which precluded verification of patient's adherence to treatment.

Despite these limitations, the main strength of our study lies in its "real-life" design. In contrast to randomized controlled clinical trials, which usually apply strict patient inclusion and exclusion criteria resulting in highly selective patient populations, real-life studies adopt pragmatic clinical care settings and procedures in non-selected patients including even those with comorbidities or concomitant medications $[31,32]$. Accordingly, the current study includes a large COPD patient population typically encountered in daily clinical practice and reflects current therapeutic approaches, thus complementing the findings of randomized controlled trials. The value of this study is further strengthened by the wide geographical representation of sites enrolling patients leading to an even more heterogeneous population representative of the general COPD population in Greece.

\section{CONCLUSIONS}

This observational study provides new data in support of tiotropium/olodaterol Respimat beneficial effects on the health and functional status in a large heterogeneous group of Greek patients suffering from COPD. More importantly, the vast majority of patients expressed preference for the Respimat device and a willingness to continue treatment with it after the end of the study.

\section{ACKNOWLEDGEMENTS}

Funding. This study was funded by Boehringer Ingelheim. Boehringer Ingelheim also funded the journal's Rapid Service Fee.

Medical Writing and Editorial Assistance. Medical writing support was provided by Maria Kyrana, Coronis Research, and funded by Boehringer Ingelheim.

Authorship. All named authors meet the International Committee of Medical Journal Editors (ICMJE) criteria for authorship for this article, take responsibility for the integrity of the work as a whole, and have given their approval for this version to be published.

Authorship Contributions. All named authors equally contributed to the study conception and design, as well as the collection, analysis, and interpretation of data. The first draft of the manuscript was written by Coronis Research and all authors commented on previous versions of the manuscript. All authors read and approved the final manuscript.

List of Investigators. The authors would like to thank all the participating investigators who enrolled patients in the study and contributed to the collection of data: Markos Merkouropoulos, Drosos Tsavlis, Konstantinos Fragkos, Panagiotis Chatziapostolou, Gerasimos Apolonatos, Athanasios Kapetagiorgis, Christos Babalis, Eleni Iliadi Charmezokova, Charalampos Papagoras, Nikolaos Chainis, Kostas Chatzakis, Maria Vounatsi, Georgios Kalfountzos, Athanasios Kiriakakis, Michalis Arvanitakis, Dimosthenis Vlaikos, Dimitris Vlachopoulos, Grigoris Georgoudakis, Alexandra Thomopoulou Katsika, Michalis Kitrou, Alexios Krokidis, Georgios Kromidas, Lemonia Mendonidou, Konstantinos Nikas, Eleni Pipini, Violeta Talou, Chrisoula Trampari, Prodromos Chatzivlasiou, Antonis Christopoulos, Anna Kontogianni, Maria Kouri, Konstantinos Perifanos, Konstantinos Christou, Dimosthenis Papapetrou, Christina Leontaridi, Makrina Michailidou Tsagari, Nikos Aggelis, Athina 
Antonaropoulou, Christos Vasiliou, Martha Gaki, Paschalis Kakavelas, Nikos Karatzas, Dimitrios Lekkos, Georgios Balasoulis, Evangelia Papageorgiou, Dionisis Papalexatos, Apostolos Pappas, Ioanna Roulia, Despoina Saatzoglou, Eleni Tzortzaki, Theodora Chatziandreou, Zoi Douflia, Christos Dainas, Andreas Dimoulis, Sofia Kalampoka, Evgenia Karianou, Alexandra Koutsokera, Christianna Konstantellou, Ioanna Kostara, Aggelos Nikitas, Ilias Tselepis, Alexandros Alexiadis, Theocharis Nikoloudakis, Charalampos Tsitsaras, Evaggelos Theodoropoulos, Panagiota Karidi, Dimitris Fraggoulakis, Agisilaos Dervas, Athina Tsouliaga, Panagiotis Giannikos, Stefania Katsoulidi, Giannis Politis, Stavros Trifon, Thomas Vardoulakis, Ioanna Tsiokou, Maria - Anna Siganaki, Dimitrios Vassalos, Mata Moshakou, Maria Kasiola, Eleni Mihelaki, Stamatia Retsou, Konstantina Pandi, Sofia Dimopoulou, Ioanna Papanikolaou, Eleni Stavrou, Kamelia Loumnitsa-Vasileskou, Ioannis Lampaditis, Christos Tzolidis, and Stiliani Mitilineou.

Disclosures. Epaminondas Kosmas and Iraklis Titopoulos have nothing to disclose. Georgios Patentalakis and Nikos Nikas are employees of Boehringer-Ingelheim Ellas.

Compliance with Ethics Guidelines. The study was conducted in compliance with the principles laid down in the Declaration of Helsinki of 1964 and its later amendments and as per local regulatory requirements, with approval obtained by the Ethics Committee of Interbalkan Hospital of Thessaloniki and the Ethics Committee of Metropolitan Hospital of Athens. Written informed consent was obtained from all individual participants included in the study.

Data Availability. The datasets generated during and/or analyzed during the current study are available from the corresponding author on reasonable request.

Open Access. This article is licensed under a Creative Commons Attribution-NonCommercial 4.0 International License, which permits any non-commercial use, sharing, adaptation, distribution and reproduction in any medium or format, as long as you give appropriate credit to the original author(s) and the source, provide a link to the Creative Commons licence, and indicate if changes were made. The images or other third party material in this article are included in the article's Creative Commons licence, unless indicated otherwise in a credit line to the material. If material is not included in the article's Creative Commons licence and your intended use is not permitted by statutory regulation or exceeds the permitted use, you will need to obtain permission directly from the copyright holder. To view a copy of this licence, visit http://creativecommons.org/licenses/by$\mathrm{nc} / 4.0 /$.

\section{REFERENCES}

1. Rhee CK, Yoshisue H, Lad R. Fixed-dose combinations of long-acting bronchodilators for the management of COPD: global and Asian perspectives. Adv Ther. 2019;36:495-519.

2. Gulart AA, Munari AB, De QAPA, et al. Does the COPD assessment test reflect functional status in patients with COPD? Chron Respir Dis. 2017;14: 37-44.

3. Ferguson G, Karpel J, Bennett N, et al. Effect of tiotropium and olodaterol on symptoms and patient-reported outcomes in patients with COPD: results from four randomised, double-blind studies. npj Prim Care Respir Med. 2017;27:7.

4. Tashkin DP, Ferguson GT. Combination bronchodilator therapy in the management of chronic obstructive pulmonary disease, http://www. goldcopd.org.

5. Global Initiative for Chronic Obstructive Lung Disease. GLOBAL STRATEGY FOR THE DIAGNOSIS, MANAGEMENT, AND PREVENTION OF CHRONIC OBSTRUCTIVE PULMONARY DISEASE 2017 REPORT Visit the GOLD website at www.goldcopd. org (C) 2017 Global Initiative for Chronic Obstructive Lung Disease, www.goldcopd.org.

6. Blair HA. Tiotropium/olodaterol: a review in COPD. Drugs. 2019;79:997-1008.

7. Russell REK. What does the TOVITO programme tell us about how we can manage COPD? Turk Thorac J. 2018;19:216-9. 
8. Miravitlles M, Urrutia G, Mathioudakis AG, et al. Efficacy and safety of tiotropium and olodaterol in COPD: a systematic review and meta-analysis. Respir Res. 2017;18:196.

9. Buhl R, de la Hoz A, Xue W, et al. Efficacy of tiotropium/olodaterol compared with tiotropium as a first-line maintenance treatment in patients with COPD who are naive to LAMA, LABA and ICS: pooled analysis of four clinical trials. Adv Ther. 2020;37:4175-89.

10. Buhl R, Singh D, de la Hoz A, et al. Benefits of tiotropium/olodaterol compared with tiotropium in patients with COPD receiving only LAMA at baseline: pooled analysis of the TONADO ${ }^{\circledR}$ and OTEMTO® studies. Adv Ther. 2020;37:3485-99.

11. Maltais F, de la $\mathrm{Hoz}$ A, Casaburi R, et al. Effects of tiotropium/olodaterol on activity-related breathlessness, exercise endurance and physical activity in patients with COPD: narrative review with meta-/ pooled analyses. Adv Ther. 2020;38:1-19.

12. Rabe KF, Chalmers JD, Miravitlles M, et al. Tiotropium/olodaterol delays clinically important deterioration compared with tiotropium monotherapy in patients with early COPD: a post hoc analysis of the TONADO ${ }^{\circledR}$ trials. Adv Ther. 2020;38:1-15.

13. Wedzicha JA, Buhl R, Singh D, et al. Tiotropium/ olodaterol decreases exacerbation rates compared with tiotropium in a range of patients with COPD: pooled analysis of the TONADO ${ }^{\circ} / D Y N A G I T O \AA$ trials. Adv Ther. 2020;37:4266-79.

14. Buhl R, Maltais F, Abrahams R, et al. Tiotropium and olodaterol fixed-dose combination versus mono-components in COPD (GOLD 2-4). Eur Respir J. 2015;45:969-79.

15. Singh D, Ferguson GT, Bolitschek J, et al. Tiotropium + olodaterol shows clinically meaningful improvements in quality of life. Respir Med. 2015;109:1312-9.

16. Chrystyn H, Small M, Milligan G, et al. Impact of patients' satisfaction with their inhalers on treatment compliance and health status in COPD. Respir Med. 2014;108:358-65.

17. Sauer R, Hänsel $M$, Buhl R, et al. International Journal of COPD Dovepress Impact of tiotropium + olodaterol on physical functioning in COPD: results of an open-label observational study. Int J COPD. 2016;11:891.

18. Valipour A, Tamm M, Kociánová J, et al. Improvement in self-reported physical functioning with tiotropium/olodaterol in central and eastern European COPD patients. Int J COPD. 2019;14:2343-54.
19. Steinmetz KO, Abenhardt B, Pabst S, et al. Assessment of physical functioning and handling of tiotropium/olodaterol Respimat ${ }^{\circledR}$ in patients with COPD in a real-world clinical setting. Int J COPD. 2019;14:1441-53.

20. Carone M, Pennisi A, D'Amato M, et al. Physical functioning in patients with chronic obstructive pulmonary disease treated with tiotropium/olodaterol Respimat in routine clinical practice in Italy. Pulm Ther. 2020;6:1-14.

21. Rau-Berger H, Mitfessel H, Glaab T. International journal of COPD dovepress tiotropium Respimat ${ }^{\circledR}$ improves physical functioning in chronic obstructive pulmonary disease. Int J COPD. 2010;5:367.

22. Valipour A, Avdeev S, Barczyk A, et al. Therapeutic success of tiotropium/olodaterol, measured using the clinical COPD questionnaire (CCQ), in routine clinical practice: a multinational non-interventional study. Int J Chron Obstruct Pulmon Dis. 2021;16:615-28.

23. Van Isselt EFVD, Spruit M, Groenewegen-Sipkema $\mathrm{KH}$, et al. Health status measured by the Clinical COPD Questionnaire (CCQ) improves following post-acute pulmonary rehabilitation in patients with advanced COPD: a prospective observational study. Npj Prim Care Respir Med. 2014;24:14007.

24. Papadopoulos G, Vardavas CI, Limperi M, et al. Smoking cessation can improve quality of life among COPD patients: validation of the clinical COPD questionnaire into Greek. BMC Pulm Med. 2011;11:13.

25. Kozma CM, Slaton TL, Monz BU, et al. Development and validation of a patient satisfaction and preference questionnaire for inhalation devices. Treat Respir Med. 2005;4:41-52.

26. Hodder R, Price D. Patient preferences for inhaler devices in chronic obstructive pulmonary disease: experience with Respimat soft mist inhaler. Int J Chron Obstruct Pulmon Dis. 2009;4:381-90.

27. Kocks JWH, Tuinenga MG, Uil SM, et al. Health status measurement in COPD: the minimal clinically important difference of the clinical COPD questionnaire. Respir Res. 2006;7:62.

28. Hanada S, Wada S, Ohno T, et al. Questionnaire on switching from the tiotropium HandiHaler to the Respimat inhaler in patients with chronic obstructive pulmonary disease: changes in handling and preferences immediately and several years after the switch. Int J COPD. 2015;10:69-77.

29. Ferguson GT, Dalby RN. Clinical implications of the tiotropium/olodaterol inhaler for patients with 
chronic obstructive pulmonary disease. Postgrad Med. 2018;130:515-22.

30. OECD. OECD guidelines on measuring subjective well-being. OECD; 2013.

31. Saturni S, Bellini F, Braido F, et al. Randomized controlled trials and real-life studies. Approaches and methodologies: a clinical point of view. Pulm Pharmacol Ther. 2014;27:129-38.

32. Mahajan R. Real world data: additional source for making clinical decisions. Int J Appl Basic Med Res. 2015;5:82. 